\title{
Investigating the Double-Peak Phenomenon in Oral Pharmacokinetics of Eurycoma Longifolia quassinoids in Lipid-Based Solid Dispersion
} Ma HQ, Ebrahimi F, Khan NAK and Chan KL

\author{
School of Pharmaceutical Sciences, Universiti Sains Malaysia, 11800, Minden, Penang, Malaysia
}

${ }^{*}$ Corresponding author: Chan KL, School of Pharmaceutical Sciences, Universiti Sains Malaysia, 11800 Minden, Penang, Malaysia, Fax: +604 6576836, Tel: +604 6532696, E-mail: kitlamc@gmail.com

Citation: Ma HQ, Ebrahimi F, Khan NAK, Chan KL (2015) Investigating the Double-Peak Phenomenon in Oral Pharmacokinetics of Eurycoma Longifolia quassinoids in Lipid-Based Solid Dispersion. J Bioequiv 1(1): 105. doi: 10.15744/2575-551X.1.105

Received Date: July 29, 2015 Accepted Date: September 01, 2015 Published Date: September 03, 2015

\begin{abstract}
$13 a(21)$-Epoxyeurycomanone (EP) and eurycomanone (EN), the polar quassinoids of Eurycoma longifolia extract (TAF2) formulated as a lipid-based solid dispersion (TAF2-SD) to increase sperm count for oligospermic infertility, displayed improvement of oral pharmacokinetics and bioavailability in rats over the non-formulated TAF2 extract. The plasma concentration-time curves of the quassinoids in both preparations displayed double-peak phenomenon, similarly observed in BCS Class 3 drugs that are highly hydrophilic and of low phospholipid membrane permeability, which therefore prompted investigation of the contributing factors. The results following the oral co-administration of TAF2-SD with the gastrointestinal transit markers indicated that less than 50\% $\mathrm{w} / \mathrm{w}$ of EP and EN were absorbed in the rat small intestine prior to $2.5 \mathrm{~h}$, the gastric emptying time of $50 \% \mathrm{w} / \mathrm{w}$ theophylline marker absorption. More than $70 \% \mathrm{w} / \mathrm{w}$ of the quassinoids were absorbed in the colon after $8 \mathrm{~h}$, the detection of $50 \% \mathrm{w} / \mathrm{w}$ sulfapyridine, the hydrolyzed metabolite of sulfasalazine marker. The non-formulated TAF2 extract at $0.028 \mathrm{ppm}$ and its major quassinoid, EN at 0.005 ppm reduced acetylcholine-and histamine-induced contractions of the guinea pig ileum, suggesting that the muscle relaxant effect contributed to the delay in gastric-emptying and absorption in the intestine. In conclusion, the formulated quassinoids improved the oral bioavailability without changing the double-peak pharmacokinetic profile, and reduced gut motility causing delayed gastric emptying and intestinal absorption.
\end{abstract}

Keywords: Standardized Eurycoma longifoia extract; Lipid-based solid dispersion; Oral pharmacokinetics and bioavailability; Double peak phenomenon

\section{Introduction}

Eurycoma longifolia Jack (E. longifolia), a small tree belonging to Simaroubaceae family, is known as 'Tongkat Ali' in Malaysia [1]. The root contains bitter-taste bioactive quassinoids, eurycomanone (EN) and 13a(21)-epoxyeurycomanone (EP) (Figure 1) that were reported to possess anti-malarial [2], anti-parasitic [3] and anti-proliferative [4] properties. Our earlier studies reported that these quassinoids in a standardized E. longifolia extract (TAF2) significantly improved the sperm count of male rats [5] and may be potentially useful for development against oligospermic infertility. However, the rat oral bioavailability of EP and EN in TAF2 was very low [6] and their plasma concentration-time curves displayed double peak phenomenon. Many lipid-soluble drugs have low oral bioavailability due to P-glycoprotein (P-gp) efflux and/or P450 metabolism especially the isoform CYP3A4 in the liver [7]. The potential substrates for P-gp efflux usually possess high log P values exceeding 2.92 [8] or between 3.6 and 4.5 [9]. In oral drug metabolism, the CYP3A4 substrates are relatively lipophilic with high log P values [10] important for P450 enzyme-substrate selectivity and binding affinity [11]. In contrast, polar compounds with low log $\mathrm{P}$ values have low binding affinity to cytochrome P450 enzymes and P-gp efflux $[8,11,12]$. Our recent unpublished data on the in vitro P-gp efflux involving TAF2 alone and in combination with inhibitor markers, verapamil and quinidine using the rat everted gut sac method displayed no significant difference in the quassinoid concentration absorbed. In addition, the in vitro metabolic studies of the rat liver homogenates and NADPH at $37{ }^{\circ} \mathrm{C}$ for $50 \mathrm{~min}$ with TAF2 or the positive controls, verapamil and quinidine also showed no metabolic changes of EP and EN in HPLC chromatogram. Thus, the low bioavailability was not caused by P-gp efflux and P450 metabolism of the quassinoids in TAF2. EP and EN, being highly water soluble and low in lipid permeability (low log P values) resembled the Biopharmaceutical Classification System (BCS) Class 3 drugs such as cimetidine and ranitidine [13,14] which also displayed double-peak pharmacokinetic plasma concentration-time curves $[15,16]$. Other contributing factors to the double peak phenomenon might include the enterohepatic recirculation and site-specific absorption [17], or bile salt interaction [18]. Delay in gastric emptying, variable gastrointestinal absorption, food effect and intestinal lymphatic transport can also cause the development of double peaks in the plasma concentration-time curves of the drugs [16,19,20]. An optimized lipid-based solid dispersion (TAF2SD) was therefore developed in the present study to improve the oral gastrointestinal absorption and bioavailability. 


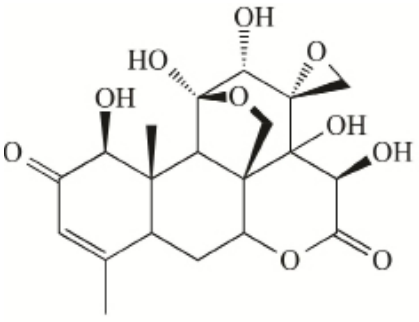

$13 \alpha(21)$-Epoxyeurycomanone (EP)

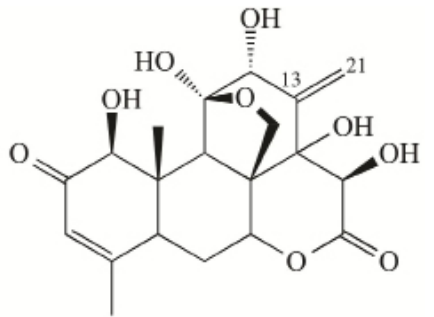

Eurycomanone (EN)

Figure 1: Chemical structures of 13a(21)-epoxyeurycomanone (EP) and eurycomanone (EN)

The primary objectives were therefore to compare the oral pharmacokinetics of E. longifolia quassinoids (EP and EN) in the formulated TAF2-SD and non-formulated TAF2 extract, and the secondary objectives were to reveal the major mechanisms causing the double-peak phenomenon in the plasma concentration-time curves of the quassinoids.

\section{Materials and Methods}

\section{Plant material and chemicals}

Eurycoma longifolia roots were purchased from Biotropics Malaysia Berhad, Kuala Lumpur, Malaysia. The numbered voucher specimens were deposited at the Penang Botanical Garden, Penang, Malaysia. Theophylline, sulfasalazine and sulfapyridine were purchased from Sigma Chemicals (St Louis, MO, USA). The analytical grade of acetonitrile for high-performance liquid chromatography (HPLC) analysis was purchased from Merck (Darmstadt, Germany).

\section{Preparation and fractionation of $E$. longifolia extract}

The $5 \mathrm{~kg}$ dried powdered roots of E. longifolia were Soxhlet-extracted (Duran, Germany) with 95\% fresh methanol (GR, Merck, Germany) for 8 hours $\times 5$ days consecutively at $60^{\circ} \mathrm{C}$. The extracts were combined, filtered and evaporated to dryness in vacuo. The residue ( $200 \mathrm{~g}, 4 \% \mathrm{w} / \mathrm{w}$ ) was dissolved in methanol and then passed through a resin column of Diaion ${ }^{\circledR} \mathrm{HP} 20$ (Mitsubishi, Japan) and eluted by gradient mixtures of increasing ratio of methanol in water (0:100-100:0) to afford four fractions (TAF1, TAF2, TAF3 and TAF4 [21]. The TAF2 solution(methanol:water = 30:70) was selected for evaporation to dryness in vacuo. The powdered residues $(30 \mathrm{~g}, 15 \% \mathrm{w} / \mathrm{w})$ were sieved to particle sizes of 125-180 $\mu \mathrm{m}$. The reference quassinoid markers of eurycomanone (EN) and $13 a(21)$-epoxyeurycomanone (EP) were isolated and purified from the TAF2 extract following previously described protocol [22]. The spectroscopic data (UV, IR, MS and NMR) of the two quassinoids were similar to those previously reported [23]. The purity of the quassinoids was above $95 \%$ as determined by a Waters Delta Prep HPLC system equipped with a Waters 2996 photodiode array detector (PDA) and Empower 2 workstation (Waters, Milford, MA).

\section{Preparation of the lipid-based solid dispersion TAF2-SD}

The optimized formulation was developed from an orthogonal array L9 $\left(3^{4}\right)$ design [24], which has advantages in rapid characterization and optimization of complicated conditions with fewer number of experiments [25,26]. The optimized protocol was then followed as shown below.

Gelucire G44/14 (Gattefossé, France) and Span 60 (Sigma Aldrich, MO, USA) at a mixture ratio of 2:1 w/w were heated and stirred until fully melted for one hour at $60{ }^{\circ} \mathrm{C}$. TAF2 was next added gradually to the molten mixture to afford a ratio mixture of $1: 3 \mathrm{w} / \mathrm{w}$, respectively with continued stirring for another $20 \mathrm{~min}$ at the same temperature [27]. The molten mixture of Gelucire G44/14 + Span $60+$ TAF2 was maintained at $60{ }^{\circ} \mathrm{C}$ for $30 \mathrm{~min}$ with continued stirring for another 10 min at room temperature until the mixture formed a semi-solid TAF2-SD.

\section{HPLC analysis}

The analysis of EP and EN was performed following the validated HPLC method previously described [6,28], with slight modifications. Briefly, $50 \mu \mathrm{L}$ of treated rat plasma was deproteinized with $2.5 \mu \mathrm{L}$ of $70 \%$ perchloric acid and then centrifuged at $10,000 \mathrm{rpm}$. The supernatant layer was separated, next added into a $200 \mu \mathrm{L}$ insert housed inside a 2-mL Agilent vial and then stored in a tray inside an Agilent 1120 Compact Autosampler at $25{ }^{\circ} \mathrm{C}$ prior to injection into the Agilent Technologies 1120 compact LC system (Agilent Technologies, Waldbronn, Germany). Each sample of $20 \mu \mathrm{L}$ was injected automatically into the LC system, comprising a guard column (Zorbax SB-C18, $4.6 \mathrm{~mm}$ i.d. $\times 12.5 \mathrm{~mm}, 5 \mu \mathrm{m}$, Agilent Technologies, Santa Clara, CA, USA) preconnected to a TC-C18 column (Agilent Technologies, Santa Clara, CA, USA, $4.6 \mathrm{~mm}$ i.d. $\times 250 \mathrm{~mm}, 5 \mu \mathrm{m}$ ) linking to an ultraviolet detector. The isocratic mobile phase consisted of acetonitrile-water (1:9) passing through the column at a flow-rate of $0.9 \mathrm{~mL} \mathrm{~min}^{-1}$. The detection was adjusted to $238 \mathrm{~nm}$ for quantitative analysis of reference markers EP and EN, and their plasma concentration [23], whereas the reference markers of theophylline and sulfapyridine in plasma were measured at $272 \mathrm{~nm}$ [28]. The chromatograms of the quassinoids were analyzed for peak height using EZChrom Elite Compact version 3.3.2 software (Scientific Software, Agilent Technologies). 


\section{HPLC Method validation}

The standard calibration curves of peak height against each quassinoid concentration were determined for six concentrations with each derived from the mean \pm SD of five replicates measurement. The linear regression line equation for each quassinoid was determined using GraphPad Prism 5 (GraphPad Software, La Jolla, CA, USA). Five concentrations with each derived from mean \pm SD of five replicates, were performed to validate the precision and accuracy for within-day and five days of between-day analysis. The recovery value of EP and EN was calculated as a percentage of actual concentration obtained from the calibration curve over the theoretical concentration. The limit of detection (LOD) and the limit of quantification (LOQ) were measured through consecutive injections of increasing concentration of the marker quassinoid solution and determination of the relative standard deviation (\% RSD) changes in peak height. The first detected concentration of $\leq 20 \%$ RSD was defined as LOD, whereas that of $\leq$ $10 \%$ was LOQ [29].

\section{The animals}

Male Sprague-Dawley rats weighing 260 -300 g were purchased from the Animal Research and Service Centre (ARASC), Universiti Sains Malaysia, Penang, Malaysia. The animals were acclimatized for 1 week in a transit room with a 12 h light/12 dark cycle for 1 week prior to onset of experiments. The animals were fed with a commercial pellet diet (Gold Coin, Penang, Malaysia) and drinking water was provided ad libitum. The rats were fasted for 12 hours prior to blood sample collection. Food was only allowed after final blood sampling. All animal experiments were humanely conducted and the protocol was approved by the Institutional Animal Ethics Committee, Universiti Sains Malaysia [USM/Animal Ethics Approval/2012/(79)(388) and USM/Animal Ethics Approval/2011/(73)(338)].

\section{Everted gut sac experiment}

The membrane permeabilities of the quassinoids in the formulated TAF2-SD and non-formulated TAF2 were compared following the intestinal absorption of EP and EN using the isolated rat everted gut sac model [30]. Eight male SD rats were euthanized using carbon dioxide. The small intestine was freshly excised into three segments of $3-4 \mathrm{~cm}$ length, washed with saline $(0.9 \% \mathrm{NaCl}$ solution), immediately kept at $37{ }^{\circ} \mathrm{C}$ in medium 199 (M 199) (M3769-1L, SIGMA, USA), and bubbled with a gas mixture of $\mathrm{O}_{2} /$ $\mathrm{CO}_{2}$ (95:5) at $\mathrm{pH}$ 7.5. The gut sac method consisted of an excised rat small intestine that was gently everted to expose the mucosal layer inside out and then tied closely at one end with a cotton thread. The other end was also tied to a 5 -cm cannula, and then filled with $0.5 \mathrm{~mL}$ of oxygenated tissue culture medium (M199) at $37{ }^{\circ} \mathrm{C}$. The gut sac was then placed inside a $25 \mathrm{~mL}$ universal bottle containing $20 \mathrm{~mL}$ of oxygenated $\mathrm{M} 199$ at $37^{\circ} \mathrm{C}$. The test sample was added into the mucosal side and incubated for 50 min (the time needed by the tissue to reach full viability and function). The integrity and viability of the gut sac was validated following the method of Wilson and Wiseman (1954) [31] involving the measurement of D-glucose concentration in both serosa and mucosa surfaces, using a glucose assay kit (SIGMA, GAGO 20-kit, USA). After incubation, the sac was washed in $0.9 \%$ saline, blotted dry, weighted and cut open. The serosa fluid was collected and the surface area of the gut sac measured. The amount of EP and EN from the formulated TAF2-SD or the non-formulated TAF2 in the serosa fluid was determined by HPLC analysis. The absorption of EP and EN was determined using Equation 1.

Percentage uptake after $50 \mathrm{~min}(\%)=$ [quassinoid $(\mu \mathrm{g})$ in the serosa side / initial amount of the quassinoid ( $\mu \mathrm{g})$ in the mucosal side] x $100 \div$ surface area of gut sac (Equation 1).

\section{Pharmacokinetic and bioavailability experiment}

Nine rats were divided equally into 3 groups following a three-way crossover study design. The animals in Group 1 were given 5 mg kg-1 of TAF2 in normal saline intravenously. The Group 2 and 3 animals were each orally administered $100 \mathrm{mg} \mathrm{kg}^{-1}$ of TAF2 and TAF2-SD (containing $100 \mathrm{mg} \mathrm{kg}^{-1}$ of TAF2), respectively in deionized $\mathrm{H}_{2} \mathrm{O}$, using an oropharyngeal cannula. After two weeks of washout period, the animals from Group 1 and 2 were orally administered with TAF2 and TAF2-SD, respectively, while those in Group 3 were administrated with TAF2, intravenously. Following another two-week washout period thereafter, the animals in Group 1 were orally administered with the TAF2-SD; Group 2 received TAF2 intravenously and Group 3 animals were orally administrated with TAF2. About $0.2 \mathrm{~mL}$ of blood sample was removed from the tail vein and kept in a heparinized micro-centrifuge tube following the intervals of $0,0.5,1,1.5,2,2.5,3,3.5,4,6,8,10$, and $24 \mathrm{~h}$ for the group given intravenous administration. For the oral-administered groups, similar blood volume was taken at intervals of $0,0.5,1,1.5,2,3,4,6,8,12$, and $24 \mathrm{~h}$. Each blood sample was centrifuged and the supernatant plasma was kept in the freezer at $-80^{\circ} \mathrm{C}$ prior to HPLC analysis of EP and EN.

\section{Data analysis}

The pharmacokinetic data comprising the maximum concentration of the quassinoids in blood $\left(\mathrm{C}_{\max }\right)$, the time at $\mathrm{C}_{\max }\left(\mathrm{t}_{\max }\right)$, the halflife of absorption $\left(\mathrm{t}_{1 / 2}\right)$ and the area under the concentration-time curve from zero to $24 \mathrm{~h}\left(\mathrm{AUC}_{0-24}\right)$ were derived from calculations using the Kinetica 5.0 software (Thermo Fisher Scientific, Waltham, MA, USA). The oral absolute and relative bioavailability of EP and EN in TAF2 and TAF2-SD were also determined. The pharmacokinetic data of oral administration of EP and EN in TAF2 and TAF2-SD were calculated using a non-compartment model, whereas the parameters for intravenous administration were calculated using two-compartment model using Kinetica 5.0 (Thermo Fisher Scientific, Waltham, MA, USA). 


\section{Statistical analysis}

The results were expressed as mean \pm SD for the pharmacokinetic data of EP and EN obtained after oral administration of TAF2 and TAF2-SD. The means of pharmacokinetic data were analyzed using $t$-test (Kinetica 5.0, Thermo Fisher Scientific, USA). The values of $P<0.05$ were considered as statistically significant.

\section{Double peak phenomenon investigation}

For determining the gastrointestinal absorption sites of the quassinoids in the formulated TAF2-SD, theophylline was selected as the gastric emptying transit marker [32], whereas the intestinal absorption was estimated by sulfasalazine, a well-established intestinal transit marker [33]. The orally administered sulfasalazine is hydrolyzed by azoreductase in the microflora around the caecum to sulfapyridine and 5-aminosalicylic acid. The appearance of sulfapyridine in the blood indicated that it has reached the caecum after oral dosing [34]. Thus, the analysis of theophylline at 50\% concentration can be compared with the measurement time of $50 \%$ quassinoid emptying from the stomach into the small intestine. The measurement of the sulfapyridine at $50 \%$ concentration provides the time estimation of 50\% quassinoid concentration reaching the colon [35]. The formulated TAF2-SD comprising 200 mg kg-1 of TAF2 (containing half concentration of EP and EN in $100 \mathrm{mg} \mathrm{kg}^{-1}$ of TAF2 selected for the pharmacokinetic studies) was mixed with theophylline $\left(20 \mathrm{mg} \mathrm{kg}^{-1}\right)$ and sulfasalazine $\left(20 \mathrm{mg} \mathrm{kg}^{-1}\right)$, accurately weighed, dissolved in deionized water and then administered to nine male rats. The volume for oral administration was restricted to $1 \mathrm{~mL} \mathrm{~kg}^{-1}$ of body weight. Blood samples of 0.2 $\mathrm{mL}$ each were collected from the tail vein into heparinized micro-centrifuge tubes after oral administration at intervals of $0,0.5,1$, $1.5,2,3,4,6,8,10,24$ and $30 \mathrm{~h}$ and then kept in the freezer at $-80^{\circ} \mathrm{C}$ prior to the HPLC analysis. The pharmacokinetic data of EP and EN obtained after oral administration of TAF2-SD followed the calculation previously described. The percentages of theophylline and sulfapyridine absorbed in the gastrointestinal tract were calculated following the Wagner-Nelson method [36].

\section{Isolated guinea pig ileum experiment}

Four adult guinea pigs weighing 300-500 g were euthanized using $\mathrm{CO}_{2}$ asphyxiation. The abdominal cavity was immediately opened and the ileum was cut at 6-in distance from the caecum. The ileum content was washed out using Tyrode's solution aerated continuously with $95 \% \mathrm{O}_{2}$ and $5 \% \mathrm{CO}_{2}$. The Tyrode's solution consisted of the following (in $\left.\mathrm{g} / \mathrm{L}\right): \mathrm{NaCl}(8), \mathrm{KCl}(0.2), \mathrm{CaCl}(0.2)$, $\mathrm{MgCl}_{2}(0.1), \mathrm{NaH}_{2} \mathrm{PO}_{4}(0.05), \mathrm{NaHCO}_{3}(1)$ and glucose (1). The sample was cut into 2.5 -cm sections and kept in aerated Tyrode's solution. The intestinal content was washed out and the specimen was mounted vertically in the organ bath, one end connected to the lower hook of the organ bath and the other one connected to a force transducer. The organ bath was filled with $18 \mathrm{~mL}$ Tyrode's solution. The ileum was equilibrated in the organ bath at the temperature of $37^{\circ} \mathrm{C}$ for 30 min before the experiment in order to obtain accurate and reproducible responses. The temperature remained at $37^{\circ} \mathrm{C}$ during the experiment. During equilibration, the bathing fluid was changed twice to avoid the accumulation of metabolic products and lack of nutrients to the tissue. The normal contraction curve was checked until the specimen becomes stable. In all experiments, the ileum was set up with an initial tension of $1 \mathrm{~g}$. A volume of $10 \mu \mathrm{L}$ of each dose was then added to the bath solution and the change of the contractive tension was recorded using a PowerLab/Chart 8 computer program (AD Instruments, Selangor, Malaysia). Agonists were in contact with the tissue for 20-25 s and applied at 2-min intervals. A dose range of $10^{-5}-100 \mathrm{ppm}$ for acetylcholine (ACh) and histamine (Hist) was tested on the ileum sample in order to select the optimized dose range. To ensure the accuracy of results, a period of 30-min incubation with $0.028 \mathrm{ppm}$ of TAF2 or $0.005 \mathrm{ppm}$ of eurycomanone was allowed before adding different doses of ACh (5-320 ppm) and Hist (48-384 ppm). The dose of eurycomanone $(0.005 \mathrm{ppm})$ was assigned following the quassinoid amount analyzed by HPLC in TAF2 extract as $17.85 \% \mathrm{w} / \mathrm{w}$. The dose-response curves of ACh and Hist were then determined in the absence and presence of TAF2 or eurycomanone.

\section{Results}

\section{HPLC Method validation}

The HPLC chromatogram showed that the peaks of EP and EN in TAF2 were well resolved to baseline separation at retention times of 18 and $24 \mathrm{~min}$, respectively. The calibration curves of EP and EN were linear $\left(r^{2}=0.9990\right.$ and 0.9999 , respectively), over a range of 0.025-5 and 0.050-10 $\mu \mathrm{g} \mathrm{mL}^{-1}$, respectively. The LOD values of EP and EN were 0.01 and $0.025 \mu \mathrm{g} \mathrm{mL} \mathrm{m}^{-1}$, respectively. The LOQ values of 0.025 and $0.05 \mu \mathrm{g} \mathrm{mL} \mathrm{m}^{-1}$ were obtained for EP and EN, respectively. For EP analysis at $0.5-10 \mu \mathrm{g} \mathrm{mL} \mathrm{m}^{-1}$, the recoveries were $100.60 \% \pm 5.14 \mathrm{RSD}$ to $104 \% \pm 2.43 \mathrm{RSD}$; its within-day accuracy and precision were $98.09 \% \pm 0.77 \mathrm{RSD}$ to $106.91 \% \pm 1.27 \mathrm{RSD}$; whereas the between-day accuracy and precision were $96.17 \% \pm 1.47$ RSD to $107.93 \% \pm 1.42$ RSD. For the analysis of EN at $0.2-10$ $\mu \mathrm{g} \mathrm{mL}{ }^{-1}$, the recoveries were $101.29 \% \pm 3.63 \mathrm{RSD}$ to $102.48 \% \pm 2.10 \mathrm{RSD}$; its within-day accuracy and precision were $97.83 \% \pm 0.43$ RSD to $108.60 \% \pm 0.19 \mathrm{RSD}$; whereas the between-day accuracy and precision were $98.14 \% \pm 1.01 \mathrm{RSD}$ to $109.88 \% \pm 0.46 \mathrm{RSD}$.

\section{Everted gut sac experiment}

The results in Figure 2 indicated that the formulation TAF2-SD significantly improved the rat intestinal membrane absorption of EP and EN by 2.87 fold $(p<0.05)$ and 2.60 fold $(p<0.01)$, respectively more than the non-formulated TAF2 extract. 
Everted gut sac membrane absorption studies $(\mathrm{n}=8)$ of EP in TAF2-SD vs. EP in TAF2

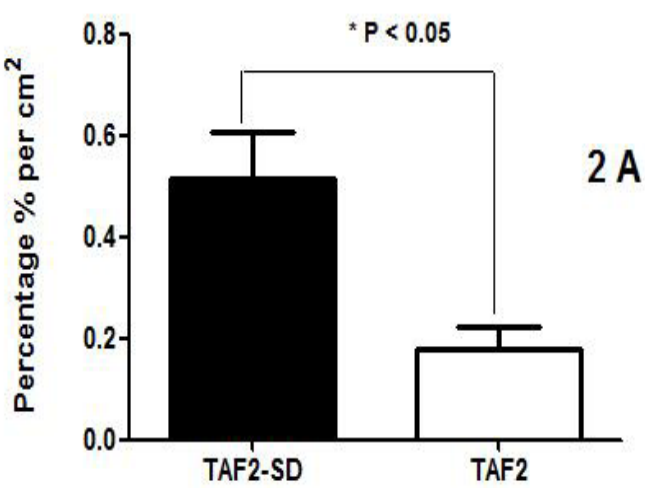

Everted gut sac membrane absorption studies $(\mathrm{n}=\mathbf{8})$ of EN in TAF2-SD vs. EN in TAF2

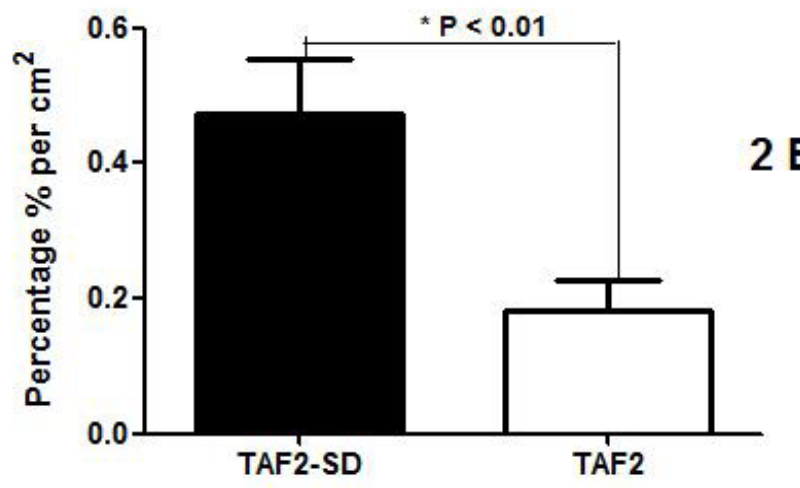

Figure 2: Absorption profiles of A: 13a(21)-epoxyeurycomanone (EP) and B: Eurycomanone (EN) in TAF2-SD solid dispersion and in TAF2 using rat everted gut sac method. Values are presented as mean \pm S.D. $(\mathrm{n}=8)$. Conditions: $20 \mathrm{~mL}, \mathrm{pH} 7.5$, medium M199, incubation time $60 \mathrm{~min} ; 37 \pm 0.5^{\circ} \mathrm{C} .^{*} \mathrm{p}$ value of less than 0.05 was considered significant

\section{Pharmacokinetics and bioavailability}

The pharmacokinetic data of EN and EP following the animals receiving TAF2 and TAF2-SD intravenously (IV) and orally are shown in Table $1 \mathrm{~A}$ and $1 \mathrm{~B}$, respectively. The rat plasma levels of both EN and EP showed a rapid decline after intravenous administration of the non-formulated TAF2 at $5 \mathrm{mg} \mathrm{kg}^{-1}$ and displayed a typical two-compartment model (Figure 3A). From the zero-time drug concentration intercepts (Table $1 \mathrm{~A})$, the A value $\left(\mu \mathrm{g} \mathrm{mL}^{-1}\right)$ of EN was about 2.9 fold higher than that of EP. However, EP $(763.80 \mathrm{~mL}$ $\left.\mathrm{kg}^{-1}\right)$ displayed about 2.6 fold higher Vc than EN $\left(295.75 \mathrm{~mL} \mathrm{~kg}^{-1}\right)$, due to EP $(65.60 \mathrm{~h})$ showing a 4 fold longer $\mathrm{t}_{1 / 2(\beta)}$ than EN $(16.22$ $\mathrm{h})$. In the oral pharmacokinetic studies, the two quassinoids in the formulated and non-formulated preparations were compared and displayed double peak phenomenon in the plasma concentration-time profile (Figure 3B). A non-compartment model was therefore applied to calculate the pharmacokinetic data (Table 1B). The $\mathrm{T} 2_{\max }, \mathrm{C} 2_{\max }, \mathrm{t}_{1 / 2}$ and $\mathrm{AUC} \mathrm{C}_{0-24 \mathrm{~h}}$ of EN in TAF2-SD were significantly higher than non-formulated TAF2 $(\mathrm{p}<0.05)$, whilst the AUC ${ }_{0-24}$ for EP was also significantly higher in TAF2-SD ( $<<$ 0.05). In addition, the AUC of EP and EN after IV administration were $22.93 \pm 11.68$ and $25.39 \pm 13.36$, respectively.

\begin{tabular}{|c|c|c|c|c|}
\hline \multirow[t]{2}{*}{ Parameters } & \multicolumn{2}{|c|}{$13 \alpha(21)$-Epoxyeurycomanone } & \multicolumn{2}{|c|}{ Eurycomanone } \\
\hline & AVE & SD & AVE & SD \\
\hline $\mathrm{A}\left(\mu \mathrm{g} \mathrm{mL}^{-1}\right)$ & 7.99 & 3.74 & 23.23 & 12.49 \\
\hline$\alpha\left(h^{-1}\right)$ & 1.22 & 0.41 & 1.22 & 0.36 \\
\hline $\mathrm{B}\left(\mu \mathrm{g} \mathrm{mL}^{-1}\right)$ & 0.19 & 0.08 & 0.19 & 0.08 \\
\hline$\beta\left(h^{-1}\right)$ & 0.02 & 0.01 & 0.04 & 0.02 \\
\hline $\operatorname{AUC}\left(\mu \mathrm{g} \mathrm{h} \mathrm{mL} L^{-1}\right)$ & 22.93 & 11.68 & 25.39 & 13.36 \\
\hline $\mathrm{k}_{10}\left(\mathrm{~h}^{-1}\right)$ & 0.40 & 0.26 & 0.95 & 0.23 \\
\hline $\mathrm{k}_{12}\left(\mathrm{~h}^{-1}\right)$ & 0.72 & 0.12 & 0.24 & 0.16 \\
\hline $\mathrm{k}_{21}\left(\mathrm{~h}^{-1}\right)$ & 0.05 & 0.03 & 0.07 & 0.05 \\
\hline $\mathrm{t}_{1 / 2(a)}(\mathrm{h})$ & 0.60 & 0.14 & 0.56 & 0.26 \\
\hline $\mathrm{t}_{1 / 2(\beta)}(\mathrm{h})$ & 65.60 & 44.47 & 16.22 & 8.56 \\
\hline $\mathrm{Vc}\left(\mathrm{mL} \mathrm{kg}{ }^{-1}\right)$ & 763.80 & 415.38 & 295.75 & 196.27 \\
\hline $\mathrm{Cl}\left(\mathrm{mL} \mathrm{h}^{-1} \mathrm{~kg}^{-1}\right)$ & 19319.18 & 10094.37 & 6214.72 & 2872.30 \\
\hline
\end{tabular}

A and B: zero time drug concentration intercepts of biphasic disposition curve; $\alpha$ and $\beta$ : rate constants for distribution and elimination phases; $\mathrm{AUC}_{0-24}$ : area under the concentration-time curve from zero to 24 hours; $C_{\max }$ : calculated maximum concentration; $T_{\max }$ : time corresponding to $C_{\max } \cdot k_{10}$ : elimination rate constant from central compartment; $\mathrm{k}_{12}$ : rate constants for the movement of drug from central compartment to peripheral compartment; $\mathrm{k}_{21}$ : rate constants for the movement of drug from peripheral compartment to central compartment; $\mathrm{t}_{1 / 2(\alpha)}$ : half-life of distribution; $\mathrm{t}_{1 / 2(\beta)}$ : half-life of elimination; $\mathrm{t}_{1 / 2}-\mathrm{K}_{10}:$ half-life of $\mathrm{K}_{10} ; \mathrm{V}_{c}:$ volume of central compartment; and $\mathrm{Cl}$ : total body clearance

Table 1A: Pharmacokinetic parameters of $13 \alpha(21)$-epoxyeurycomanone (EP) and eurycomanone (EN) after IV administration of TAF2 $\left(5 \mathrm{mg} \mathrm{kg}^{-1}\right)$ in rats. Values are presented as mean \pm S.D. $(\mathrm{n}=9)$ 


\begin{tabular}{|c|c|c|c|c|}
\hline \multirow{2}{*}{ Fabs (\%) } & \multicolumn{2}{|c|}{$13 \boldsymbol{\alpha}(\mathbf{2 1})$-epoxyeurycomanone } & \multicolumn{2}{c|}{ Eurycomanone } \\
\cline { 2 - 5 } & TAF2 & TAF2-SD & TAF2 & TAF2-SD \\
\hline $\mathrm{T} 1_{\text {max }}(\mathrm{h})$ & $1.00 \pm 0.12$ & $0.94 \pm 0.19$ & $0.89 \pm 0.11$ & $0.83 \pm 0.14$ \\
\hline $\mathrm{T} 2_{\max }(\mathrm{h})$ & $5.13 \pm 0.48$ & $6.44 \pm 0.44$ & $4.56 \pm 0.58$ & $7.11 \pm 0.35^{*}$ \\
\hline $\mathrm{C}_{\text {max }}\left(\mu \mathrm{gLL}^{-1}\right)$ & $0.11 \pm 0.03$ & $0.16 \pm 0.05$ & $0.32 \pm 0.10$ & $0.20 \pm 0.05$ \\
\hline $\mathrm{C}_{\text {max }}\left(\mu \mathrm{g} \mathrm{mL}^{-1}\right)$ & $0.11 \pm 0.02$ & $0.17 \pm 0.04$ & $0.41 \pm 0.13$ & $0.53 \pm 0.14^{*}$ \\
\hline $\mathrm{t}_{1 / 2}(\mathrm{~h})$ & $22.67 \pm 1.61$ & $20.38 \pm 0.30$ & $17.92 \pm 1.43$ & $11.36 \pm 0.66^{*}$ \\
\hline $\mathrm{AUC}_{0-24 \mathrm{~h}}\left(\mu \mathrm{gg} \cdot \mathrm{h} \mathrm{mL}^{-1}\right)$ & $1.72 \pm 0.29$ & $2.58 \pm 0.28^{*}$ & $2.34 \pm 0.22$ & $6.25 \pm 0.88^{*}$ \\
\hline $\mathrm{F}_{\text {abs }}(\%)$ & $7.50 \pm 1.19$ & $11.25 \pm 1.67^{*}$ & $9.22 \pm 0.86$ & $24.62 \pm 3.61^{*}$ \\
\hline $\mathrm{F}_{\text {rel }}$ & \multicolumn{2}{|c|}{$1.69 \pm 0.34$} & \multicolumn{2}{|c}{$2.75 \pm 0.53$} \\
\hline
\end{tabular}

Values are presented as mean \pm S.D. $\left(\mathrm{n}=9\right.$ ). ${ }^{*}$ Significant different level at $p<0.05 ; \mathrm{Cl}_{\max }:$ maximum concentration of first peak; $\mathrm{Tl}_{\max }:$ time corresponding to $\mathrm{Cl}_{\max }$ $\mathrm{C}_{\text {max }}$ : maximum concentration of second peak; $\mathrm{T}_{\text {max }}$ : time corresponding to $\mathrm{C}_{\max }, \mathrm{t}_{1 / 2}:$ half-life of the absorption phase; $\mathrm{AUC}_{0-24}$, area under the concentration-time

Table 1B: Pharmacokinetics parameters of $13 \alpha(21)$-epoxyeurycomanone (EP) and eurycomanone (EN) for E. longifolia non-formulated TAF2 and formulated extract TAF2-SD after $100 \mathrm{mg} \mathrm{kg}^{-1}$ oral administrations

\section{$3 \mathbf{A}$}

$13 \alpha(21)$-epoxyeurycomanone

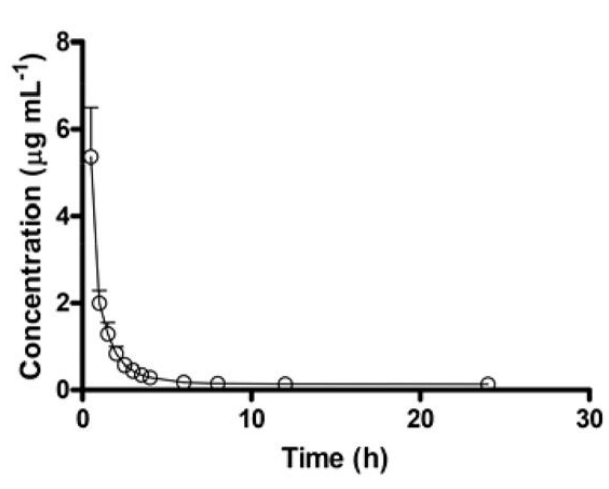

3B
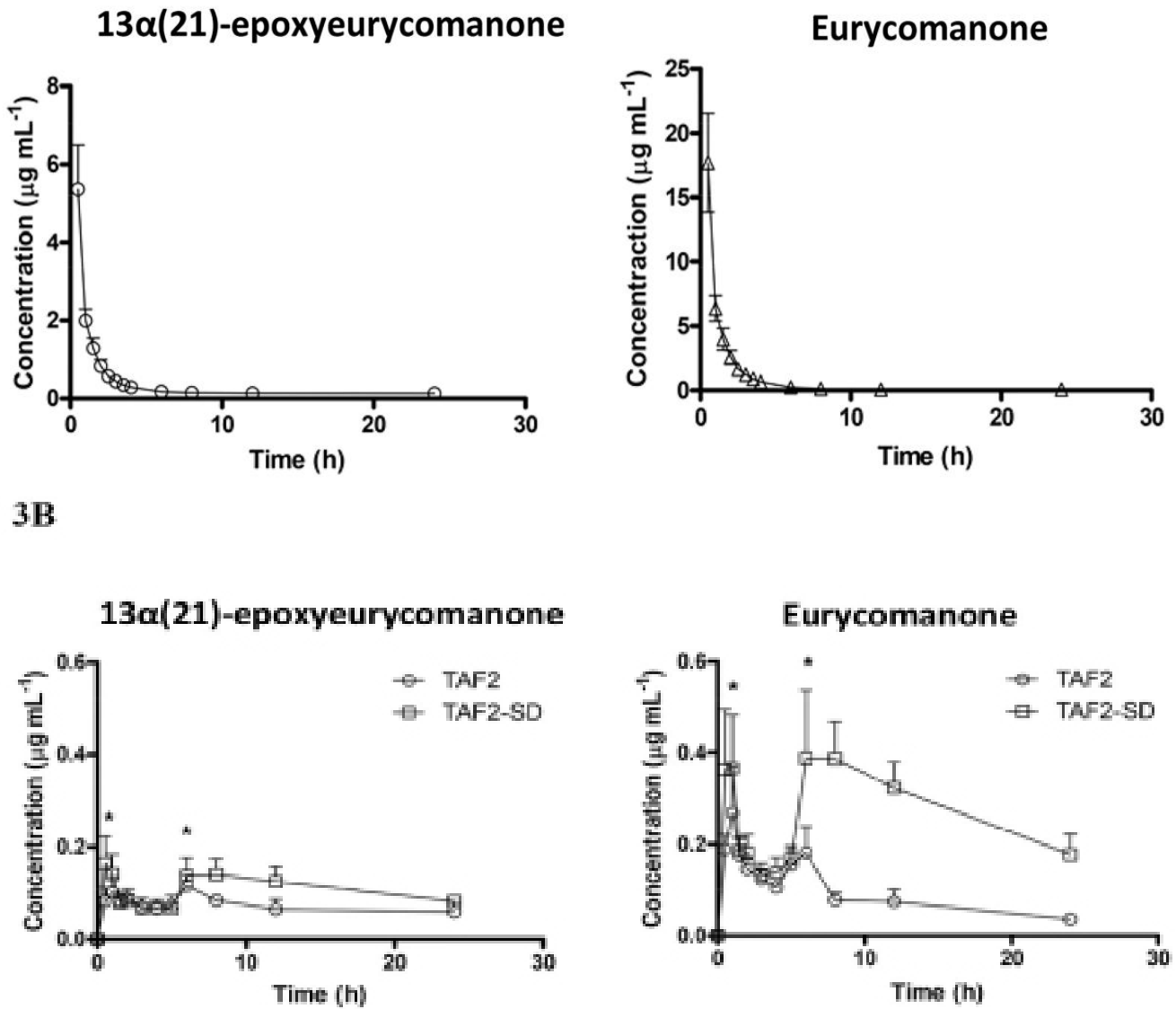

Figure 3A: Plasma concentration-time curves of $13 \alpha(21)$-epoxyeurycomanone (EP) and eurycomanone (EN)after intravenous administration of $5 \mathrm{mg} \mathrm{kg}^{-1}$ TAF2. B: Plasma concentration-time curves of EP and EN in TAF2 (100 mg kg-1) and TAF2-SD (containing 100 mg kg-1 TAF2) after a single oral-dose administration. Values are presented as mean \pm S.D. $(n=9) .^{*} \mathrm{p}$ value of less than 0.05 was considered significant

Thus, the absolute oral bioavailabilities $\left(\mathrm{F}_{\mathrm{abs}}\right)$ of EP in TAF2 and TAF2-SD were $7.50 \pm 1.19 \%$ and $11.25 \pm 1.67$, respectively, and those of EN in TAF2 and TAF2-SD were $9.22 \pm 0.86 \%$ and $24.62 \pm 3.61 \%$, respectively. The relative bioavailabilities $\left(\mathrm{F}_{\text {rel }}\right)$ of EP and EN in TAF2-SD were 1.69 and 2.75 fold, respectively, higher than in TAF2. The results also showed that the formulation improved the oral bioavailability of EN much more than of EP even though higher amount of EN was originally in the nonformulated TAF2 extract. The HPLC chromatogram of blank rat plasma, the EP and EN in the rat plasma sample at $1 \mathrm{~h}$ after oral administration of $100 \mathrm{mg} \mathrm{kg}^{-1}$ TAF2 extract and TAF2-SD (containing $100 \mathrm{mg} \mathrm{kg}^{-1} \mathrm{TAF} 2$ extract) is shown in Figure 4. 


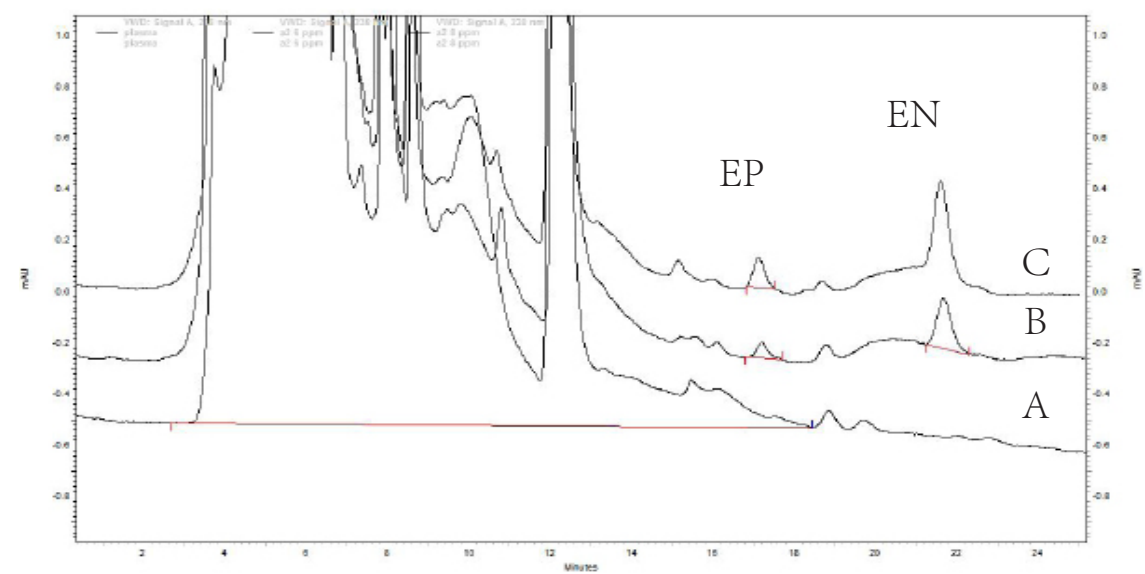

Figure 4: HPLC chromatogram of (A) Blank rat plasma and (B) the $13 \alpha(21)$-epoxyeurycomanone (EP) and eurycomanone (EN) in rat plasma at $1 \mathrm{~h}$ after oral administration of $100 \mathrm{mg} \mathrm{kg}^{-1} \mathrm{TAF} 2$ extract and (C) EP and EN in rat plasma at $1 \mathrm{~h}$ after oral administration of TAF2-SD (containing $100 \mathrm{mg} \mathrm{kg}^{-1}$ TAF2 extract). TC-C18 $(250 \mathrm{~mm} \times 4.6 \mathrm{~mm}$ i.d., $5 \mu \mathrm{M})$ column; column temperature $25^{\circ} \mathrm{C}$; mobile phase acetonitrile-water $(10: 90)$; flow rate $1.0 \mathrm{~mL} \mathrm{~min}^{-1}$; UV wavelength $238 \mathrm{~nm}$

\section{Double-peak phenomenon investigation}

A double peak phenomenon in the plasma concentration-time curves of EP and EN, was observed following oral administration of single dose TAF2 (100 mg kg-1) and TAF2-SD (containing $100 \mathrm{mg} \mathrm{kg}^{-1} \mathrm{TAF} 2$ ). (Figure 3B). The double peak pharmacokinetic profile was also observed in the combination mixture of TAF2-SD (200 mg kg-1 of TAF2 containing only half concentration of EP and $\mathrm{EN}$ in the $100 \mathrm{mg} \mathrm{kg}^{-1}$ of TAF2 selected for pharmacokinetic studies) with the gastrointestinal transit markers (Figure 5). The $200-\mathrm{mg} / \mathrm{kg}^{-1}$ TAF2-SD study on the double-peak phenomen on provided a clearer and easier plasma concentration-time profile to distinguish the transit times for $\mathrm{T}_{50}$ of theophylline and $\mathrm{T}_{50}$ of sulfapyridine.

The appearance of the first smaller peak at a shorter time $\mathrm{T} 1_{\text {max }}$ of EP and EN in TAF2-SD at $1 \mathrm{~h}$, was in the stomach before $50 \%$ w/w of marker theophylline appeared in the small intestine at $2.5 \mathrm{~h}$. A second larger broad peak of longer T2 $2_{\text {max }}$ appeared after $8 \mathrm{~h}$, the time of 50\% sulphapyridine absorption where more than $70 \%$ of the quassinoids were absorbed in the colon (Figure 5). Following the pharmacokinetics profiles of EP and EN, about $74.36 \%$ of EP and $80.27 \%$ of EN in TAF2-SD took more than $8 \mathrm{~h}$ to reach the colon. The results indicated that the colon was the main absorption site for the quassinoids following the oral administration of TAF2-SD (Figure 5).

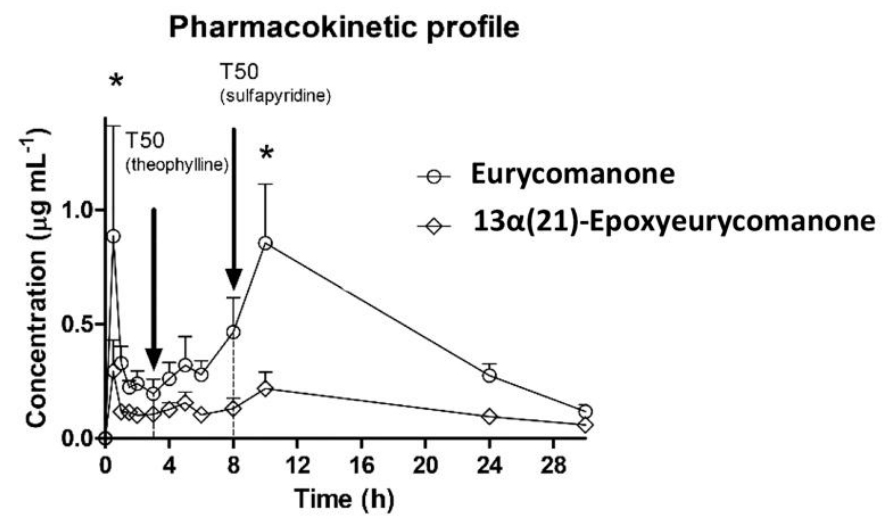

Figure5: Mean plasma concentration vs.time curves of 13a(21)-epoxyeurycomanone(EP) and eurycomanone (EN) after oral administration of $200 \mathrm{mg} \mathrm{kg}^{-1}$ TAF2-SD and distribution in the gastrointestinal tract. T50 (theophylline) and T50 (sulfapyridine) are the time for 50\% of theophylline and $50 \%$ of sulfapyridine to be absorbed respectively. Values are presented as mean \pm S.D. ( $n=9$ ). The TAF2 used was 200 mg $\mathrm{kg}^{-1}$ instead of $100 \mathrm{mg} \mathrm{kg}^{-1}$ because it contained only $50 \% \mathrm{w} / \mathrm{w}$ of EP and EN in TAF2 used for pharmacokinetic studies in Figure 3B

\section{Isolated guinea pig ileum experiment}

The dose-response of acetylcholine (ACh) at 5 to $320 \mathrm{ppm}$ induced contractions of the isolated guinea pig ileum and displayed a typical sigmoidal curve (Figure 6A and 6B). The ileum upon exposure to a 30-min incubation with 0.028 ppm of TAF2 (containing $17.85 \% \mathrm{w} / \mathrm{w}$ eurycomanone) or $0.005 \mathrm{ppm}$ of eurycomanone followed by adding different doses of ACh (5-320 ppm) caused significant reduction $(\mathrm{p}<0.05)$ in the ACh-induced contractions of the ileum. With similar sigmoidal dose-response curve was shown by histamine (Hist) at 48-384 ppm. In the presence of TAF2 at $0.028 \mathrm{ppm}$ or eurycomanone at 0.005 ppm, the dose-response curve of histamine-induced contractions of the ileum was also similarly significantly reduced $(\mathrm{p}<0.05)$. The dose assigned for eurycomanone (EN) followed the quassinoid quantity analyzed by HPLC in the TAF2 extract as $17.85 \% \mathrm{w} / \mathrm{w}$. 

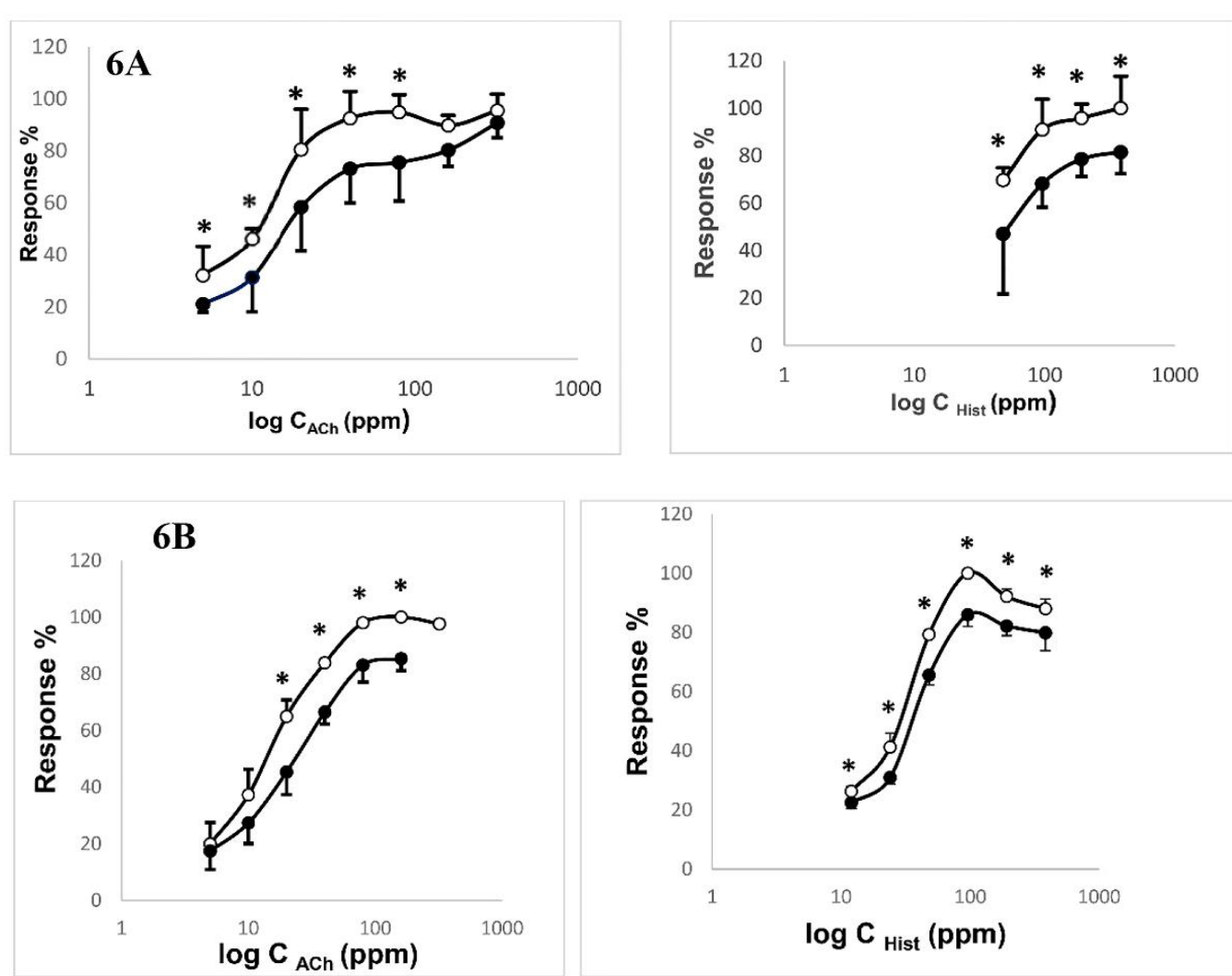

Figure 6A: The effect of TAF2 on the acetylcholine (ACh)- and histamine (Hist)-induced contractions of guinea-pig ileum, $\mathrm{n}=6$. The white circles indicate the ACh- and Hist-induced contraction, respectively. The black circles represent the effect of $0.028 \mathrm{ppm}$ TAF2 on ACh-and Hist-induced ileal contraction, respectively. B: The effect of eurycomanone on ACh- and Hist-induced contractions of guinea-pig ileum, $\mathrm{n}=4$. The white circles indicate the ACh- and Hist-induced contraction, respectively. The black circles signify the effect of $0.005 \mathrm{ppm}$ eurycomanone on ACh- and Hist-induced ileal contraction, respectively. All the results are reported basedon contractile response $\% \pm$ SD. ${ }^{*}$ Significant at $p<0.05$ (Mann-Whitney U Test)

\section{Discussion}

The standardized E. longifolia extract TAF2 formulated into a lipid-based solid dispersion significantly improved the ileum absorption of 13a(21)-epoxyeurycomanone (EP) and eurycomanone (EN) in the isolated everted rat gut experiment. From the intravenous pharmacokinetic data (Table 1A), EP displayed higher volume of distribution in the central compartment (Vc) than EN because of longer half-life of elimination $\left(\mathrm{t}_{1 / 2(\beta)}\right)$ probably due to EP being more polar than EN, thus remaining in the central compartment and distributing less into other compartments. In the oral pharmacokinetic studies, the levels of $\mathrm{AUC}_{0-24}$, the $\mathrm{F}_{\mathrm{abs}}$ and $\mathrm{F}_{\text {rel }}$ of EN and EP in the formulated TAF2-SD were higher than those of the non-formulated TAF2. Though EN $(14.49 \pm 0.26 \% \mathrm{w} / \mathrm{w})$ was about two fold higher than EP $(7.39 \pm 0.17 \% \mathrm{w} / \mathrm{w})$ in concentration from the original TAF2 extract, the $\mathrm{AUC}_{0-24}$ and $\mathrm{F}_{\text {abs }}$ data (Table 1B) showed a 3.3 fold higher increment of blood EN than EP concentrations produced by the formulated TAF2-SD. From the previously reported data, EN was relatively less polar than EP, since the latter developed a shorter retention time than EN in the reverse phase HPLC chromatogram [22], and a smaller $\mathrm{R}_{f}$ value than EN on TLC of normal phase silica gel coated plates [23]. Furthermore, the structures of both quassinoids (Figure 1) are the same except the epoxide moiety in EP, whereas EN displayed a double bond at C-13 and C-21. The epoxide of EP has localized pairs of electrons at the oxygen making the overall structure more polar than EN, which has pi electrons distributing between C-13 and C-21, instead. Despite these evidences in support of EN being relatively less polar than EP, there was no significant difference in the percentage absorption per $\mathrm{cm}^{2}$ of the ileum between the quassinoids (Figure 2). The quassinoid polarity may be one of the factors affecting absorption. The other factor, the quassinoid membrane permeability is also highly important. The higher $\log \mathrm{P}$ value of EP (-0.43) contributed to greater membrane permeability than EN (-1.46) at $\mathrm{pH} 1$ in the stomach [21]. Furthermore, the in vivo percentage absorption-time curve of EP (log $\mathrm{P}=-0.67$ at $\mathrm{pH} 7)$ appeared higher than $\mathrm{EN}(\log \mathrm{P}=-1.44$ at $\mathrm{pH} 7)$ in the small intestine before reaching the colon. Although the chemical structure indicated that EP was more polar, its lipophilicity was not lower than EN. However, when both quassinoids were formulated into a more lipid-based matrix, EN of higher concentration and lower polarity than EP in the E. longifolia extract displayed greater absolute oral bioavailability.

Trace amount of the quassinoids managed to reach the blood circulation through the stomach by close vicinity to an "absorption window" [37] and probably involving limited active transport or facilitated diffusion. Thus, the delay in stomach emptying contributed towards the establishment of the double peak phenomenon since more than $50 \%$ of the quassinoids in TAF2-SD and TAF2 took more than $2.5 \mathrm{~h}$ to reach the small intestine. 
Journal of Bioequivalence Studies

The probability of absorption through the lymphatic system may not be applicable for the polar quassinoids due to the strong hydrophilic property. In fact, the lipophilic compounds possessing log $\mathrm{P}$ value of greater than 5 can only be absorbed via the systemic lymphatic route [38]. The effect of enterohepatic recirculation contributing to the double peak phenomenon was most unlikely since double peaks were not present after intravenous administration [21] (Figure 3A). Furthermore, the food effect on absorption may also not arise since the rats were fasted until blood collection. The high water solubility and low lipid permeability of the quassinoids appeared as a plausible explanation towards the formation of double peak phenomenon since the physicochemical properties mimicked those of the BCS Class 3 drugs. When formulated in a lipid-based matrix, some of these drugs also displayed double peak phenomenon. [39]. For example, the bioavailability of polyphenols, the BCS Class III compounds has been similarly improved by lipid-based formulations [40].

The pharmacological effect of drugs can support the formation of the double peak pharmacokinetic profile. For example, the double-peak oral pharmacokinetic profile of alprazolam was related to the gut muscle relaxant effect that resulted in reduced gastric motility [41]. The pharmacological effect of TAF2 $(0.028 \mathrm{ppm})$ and its major quassinoid, EN (0.005 ppm) on acetylcholineand histamine-induced contractions of the guinea pig ileum, provided evidence that the anti-cholinergic and anti-histaminergic muscle relaxant effect of the quassinoids reduced the gut motility (Figures 6A and 6B), thereby delaying gastric-emptying and intestinal absorption (Figure 5). The lipid-based matrix of solid dispersion significantly increased the absorption of quassinoids in the colon, as lipophilic formulations were reported more permeable in the colon than the small intestine [42]. Consequently, a higher quassinoid level in the blood also resulted in greater muscle relaxant effect and reduction in gut motility, ultimately producing no change in the double-peak pharmacokinetic profile.

\section{Conclusion}

The present study showed that the lipid-based solid dispersion formulation of polar quassinoids in E. longifolia significantly improved intestinal absorption and oral bioavailability in the blood, but no change was observed for the developed double-peak pharmacokinetic profile. The anti-cholinergic and anti-histaminergic properties of the quassinoids affected the gut motility causing delayed gastric emptying and major absorption in the colon.

\section{Acknowledgement}

The authors wish to thank the Ministry of Agriculture and Agro-based Industry, Malaysia for the partial financial support given under the NKEA Research Grant Scheme No: (NRGS)-NH0711S001.

\section{References}

1. Perry LM, Metzger J (1980) Medicinal plants of East and Southeast Asia: attributed properties and uses. Cambridge: MIT Press.

2. Chan KL, Choo CY, Abdullah NR (2005) Semisynthetic 15-O-acyl- and 1,15-di-O-acyleurycomanones from Eurycoma longifolia as potential antimalarials. Planta Med 71: 967-9.

3. Jiwajinda S, Santisopasri V, Murakami A, Sugiyama H, Gasquet M, et al. (2002) In vitro anti-tumor promoting and anti-parasitic activities of the quassinoids from Eurycoma longifolia, a medicinal plant in Southeast Asia. J Ethnopharmacol 82: 55-8.

4. Wong PF, Cheong WF, Shu MH, Teh CH, Chan KL, et al. (2012) Eurycomanone suppresses expression of lung cancer cell tumor markers, prohibitin, annexin 1 and endoplasmic reticulum protein 28. Phytomedicine 19: 138-44.

5. Chan KL, Low BS, Teh CH, Das PK (2009) The effect of Eurycoma longifolia on sperm quality of male rats. Nat Prod Commun 4: 1331-6.

6. Low BS, Ng BH, Choy WP, Yuen KH, Chan KL (2005) Bioavailability and pharmacokinetic studies of eurycomanone from Eurycoma longifolia. Planta Med 71: 803-7.

7. Fasinu P, Pillay V, Ndesendo VMK, Du toit LC, Choonara YE (2011) Diverse approaches for the enhancement of oral drug bioavailability. Biopharm Drug Dispos 32: 185-209.

8. Wang RB, Kuo CL, Lien LL, Lien EJ (2003) Structure-activity relationship: analyses of P-glycoprotein substrates and inhibitors. J Clin Pharm Ther 28: 203-28.

9. Bain LJ, Leblanc GA (1996) Interaction of Structurally Diverse Pesticides with the Human MDR1Gene Product P-Glycoprotein. Toxicol Appl Pharm141: 288-98. 10. Lewis DF (2000) On the recognition of mammalian microsomal cytochrome P450 substrates and their characteristics: Towards the prediction of human $\mathrm{p} 450$ substrate specificity and metabolism. Biochem Pharmacol 60: 293-306.

11. Smith DA, Ackland MJ, Jones BC (1997) Properties of cytochrome P450 isoenzymes and their substrates Part 2: properties of cytochrome P450 substrates. Drug Discov Today 2: 479-86.

12. Lewis DF, Jacobs MN, Dickins M (2004) Compound lipophilicity for substrate binding to human P450s in drug metabolism. Drug Discov Today 9: 530-7.

13. Reddy BBK, Karunakar A (2011) Biopharmaceutics Classification System: A regulatory approach. Dissolution Technol 18: 31-7.

14. Lobenberg R, Amidon GL (2000) Modern bioavailability, bioequivalence and biopharmaceutics classification. New scientific approaches to international regulatory standards. Eur J Pharm Biopharm 50: 3-12.

15. Yin OQ, Tomlinson B, Chow AH, Chow MS (2003) A modified two-portion absorption model to describe double-peak absorption profiles of ranitidine. Clin Pharmacokinet 42: 179-92.

16. Oberle RL, Amidon GL (1987) The influence of variable gastric emptying and intestinal transit rates on the plasma level curve of cimetidine; an explanation for the double peak phenomenon. J Pharmacokinet Biopharm 15: 529-44.

17. Davies NM, Takemoto JK, Brocks DR, Yáñez JA (2010) Multiple peaking phenomena in pharmacokinetic disposition. Clin pharmacokinet 49: 351-77.

18. Miyazaki S, Inoue H, Yamahira T, Nadai T (1979) Interaction of drugs with bile components. I. Effects of bile salts on the dissolution behavior of indomethacin and phenylbutazone. Chem Pharm Bull 27: 2468-72. 
19. Piyapolrungroj N, Zhou YS, Li C, Liu G, Zimmermann E, et al. (2000) Cimetidine absorption and elimination in rat small intestine. Drug Metab Dispos 28: 65-72.

20. Jan KC, Chang YW, Hwang LS, Ho CT (2012) Tissue distribution and cytochrome p450 inhibition of sesaminol and its tetrahydrofuranoid metabolites. J Agric Food Chem 60: 8616-23.

21. Low BS, Teh CH, Yuen KH, Chan KL (2011) Physico-chemical effects of the major quassinoids in a standardized Eurycoma longifolia extract (Fr 2) on the bioavailability and pharmacokinetic properties, and their implications for oral antimalarial activity. Nat Prod Commun 6: 337-41.

22. Teh CH, Murugaiyah V, Chan KL (2011) Developing a validated liquid chromatography-mass spectrometric method for the simultaneous analysis of five bioactive quassinoid markers for the standardization of manufactured batches of Eurycoma longifolia Jack extract as antimalarial medicaments. J Chromatogr A 1218: 1861-77.

23. Low BS, Das PK, Chan KL (2014) Acute, reproductive toxicity and two-generation teratology studies of a standardized quassinoid-rich extract of Eurycoma longifolia Jack in Sprague-Dawley rats. Phytother Res 28: 1022-9.

24. Onuki Y, Morishita M, Takayama K (2004) Formulation optimization of water-in-oil-water multiple emulsion for intestinal insulin delivery. J Control Release 97: 91-9.

25. Guo L, Cho SY, Kang SS, Lee S-H, Baek H-Y, et al. (2007) Orthogonal array design for optimizing extraction efficiency of active constituents from JakyakGamcho Decoction, the complex formula of herbal medicines, Paeoniae Radix and Glycyrrhizae Radix. J Ethnopharmacol 113: 306-11.

26. Wang SC, Liao HJ, LeeWC, Huang CM, Tsai TH (2008) Using orthogonal array to obtain gradient liquid chromatography conditions of enhanced peak intensity to determine geniposide and genipin with electrospray tandem mass spectrometry. J Chromatogr A1212: 68-75.

27. Barker SA, Yap SP, Yuen KH, McCoy CP, Murphy JR, et al. (2003) An investigation into the structure and bioavailability of $\alpha$-tocopherol dispersions in Gelucire 44/14. J Control Release 91: 477-88.

28. Fujioka Y, Metsugi Y, Ogawara KI, Higaki K, Kimura T (2008) Evaluation of in vivo dissolution behavior and GI transit of griseofulvin, a BCS class II drug. Int J Pharm 352: 36-43.

29. Lobrutto R, Patel T (2006) Method Validation. HPLC for Pharmaceutical Scientists. John Wiley \& Sons, Inc.

30. Barthe L, Woodley JF, Kenworthy S, Houin G (1998) An improved everted gut sac as a simple and accurate technique to measure paracellular transport across the small intestine. Eur J Drug Metabolism Pharmacokinet 23: 313-23.

31. Wilson TH, Wiseman G (1954) The use of sacs of everted small intestine for the study of the transference of substances from the mucosal to the serosal surface. J Physiol 123: 116-25.

32. Porsgaard T, Straarup EM, Hoy CE (2003) Gastric emptying in rats following administration of a range of different fats measured as acetaminophen concentration in plasma. Ann Nutr Metab 47: 132-8.

33. Kellow JE, Borody TJ, Phillips SF, Haddad AC, Brown ML (1986) Sulfapyridine appearance in plasma after salicylazosulfapyridine. Another simple measure of intestinal transit. Gastroenterol 91: 396-400.

34. Chourasia MK, Jain SK (2003) Pharmaceutical approaches to colon targeted drug delivery systems. J Pharm Pharm Sci 6: 33-66.

35. Peh KK, Yuen KH (1996) Indirect gastrointestinal transit monitoring and absorption of theophylline. Int J Pharm 139: 95-103.

36. Sanaka M, Yamamoto T, Tsutsumi H, Abe K, Kuyama Y (2005) Wagner-Nelson method for analysing the atypical double-peaked [13CO ${ }_{2}$ ] excretion curve in the [13C]-octanoate gastric emptying breath test in humans. Clin Exp Pharmacol Physiol 32: 590-4.

37. Jamil F, Kumar S, Sharma S, Vishvakarma P, Singh L (2011) Review on stomach specific drug delivery systems: Development and evaluation. Intern J Res Pharmaceu Biomed Sci 2: 1427-33.

38. Jannin V, Musakhanian J, Marchaud D (2008) Approaches for the development of solid and semi-solid lipid-based formulations. Adv Drug Delivery Rev 60: 734-46.

39. Chang RK, Shojaei AH (2004) Effect of a lipoidic excipient on the absorption profile of compound UK 81252 in dogs after oral administration. J Pharm Sci 7: $8-12$.

40. Kidd PM (2009) Bioavailability and activity of phytosome complexes from botanical polyphenols: the silymarin, curcumin, green tea, and grape seed extracts. Altern Med Rev 14: 226-46.

41. Wang Y, Roy A, Sun L, Lau CE (1999) A double-peak phenomenon in the pharmacokinetics of alprazolam after oral administration. Drug Metab Dispos 27: 855-9.

42. Fagerholm U, Lindahl A, Lennernas H (1997) Regional intestinal permeability in rats of compounds with different physicochemical properties and transport mechanisms. J Pharm Pharmaco 149: 687-90.

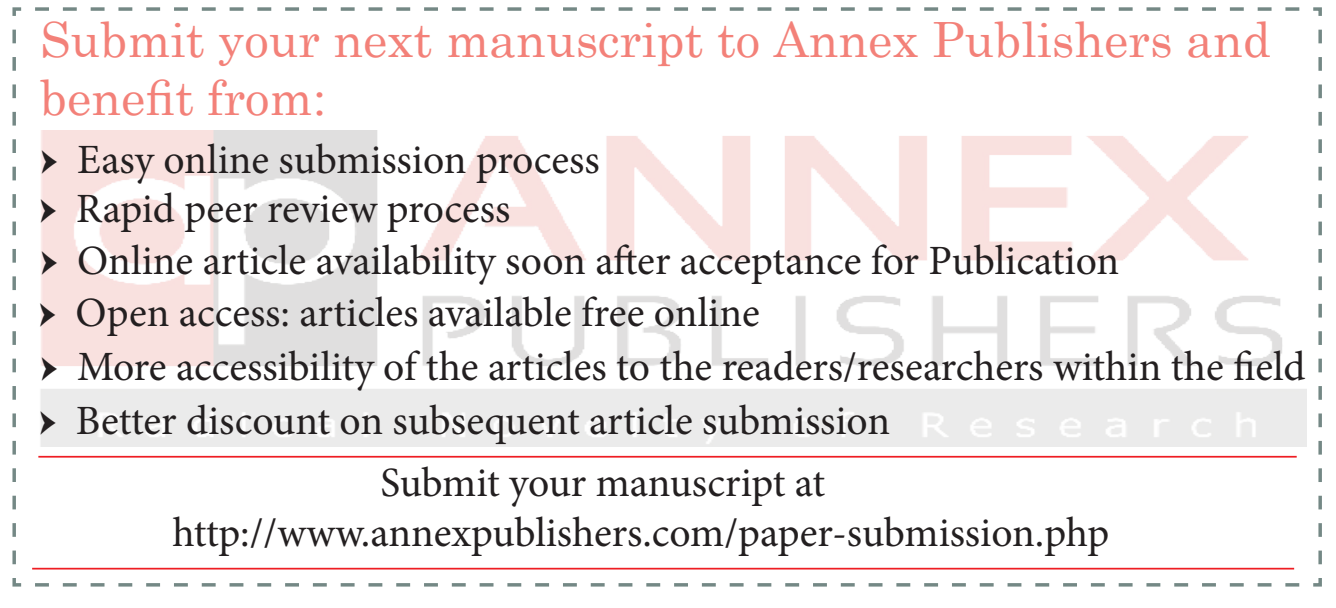

